INCIDENCIA DE LA INTELIGENCIA COMPETITIVA EN EL DESARROLLO ECONÓMICO DE LAS EMPRESAS DE LA PROVINCIA DE SANTA ELENA. 


\title{
Incidencia de la inteligencia competitiva en el desarrollo económico de las empresas de la provincia de Santa Elena.
}

\section{Impact of competitive intelligence in the economic development of enterprises in the province of Santa Elena.}

\author{
Collins Ventura Nathyn ${ }^{1}$, Soraya Linzán Rodríguez ${ }^{2}$. \\ Universidad Estatal Península de Santa Elena. Sistema de Nivelación y Admisión $\left({ }^{1}\right)$ \\ Universidad Estatal Península de Santa Elena. Facultad de Ciencias Administrativa $\left({ }^{2}\right)$ \\ Email: collinsnat 17@live.com
}

\begin{abstract}
Resumen
El objetivo de este artículo fue analizar la incidencia de la inteligencia competitiva en el desarrollo económico de las empresas. La inteligencia competitiva ayuda a las empresas a definir y conocer la industria, así como a identificar las fortalezas y debilidades de sus rivales, lo que incluye actividades de inteligencia para reunir datos sobre los competidores e interpretarlo; si se hace correctamente, ayuda a la compañía a evitar sorpresas porque se anticipan los movimientos de los competidores y se disminuye el tiempo de respuesta. Se implementó una investigación exploratoria correlacional para poder diagnosticar el desempeño organizacional de las empresas de la provincia de Santa Elena. Los métodos implementados son deductivo e inductivo, mediante técnicas de observación descriptiva, entrevistas a gerentes de las empresas y encuestas a trabajadores de las empresas para conocer la situación real. Se seleccionó 32 empresas de la provincia de Santa Elena en las que se efectuaron entrevistas a gerentes. Los resultados demuestran que, con la potenciación de fortalezas de la empresa, y por ende el aprovechamiento de oportunidades del entorno, se generará un desarrollo económico de la empresa que implemente acciones sólidas y oportunas.
\end{abstract}

Palabras clave: Inteligencia competitiva, desarrollo económico, desempeño organizacional, oportunidades del entorno, acciones estratégicas.

\begin{abstract}
The objective of this article was to analyze the incidence of competitive intelligence in the economic development of companies. Competitive intelligence helps companies define and know the industry, as well as identify the strengths and weaknesses of their rivals, which includes intelligence activities to gather data about competitors and interpret it; If done correctly, helps the company to avoid surprises because they anticipate the movements of competitors and decreases the response time. An exploratory correlational research was implemented to diagnose the organizational performance of companies in the province of Santa Elena. The methods implemented are deductive and inductive, using descriptive observation techniques, interviews with company managers and surveys of company workers to know the real situation. We selected 32 companies from the province of Santa Elena where interviews were conducted with managers. The results show that by strengthening the company's strengths, and thus taking advantage of opportunities in the environment, will generate economic development of the company that implements sound and timely actions.
\end{abstract}

Keywords: Competitive intelligence, economic, organizational performance, environmental opportunities, strategic actions. 


\section{Introducción.}

La inteligencia competitiva permite conocer e interpretar cada una de las actividades de los competidores, dando a evidenciar las debilidades que se conviertan en fortalezas y oportunidades para beneficio de la empresa. Siendo los beneficios de emplear adecuadamente la inteligencia competitiva en las empresas, obteniendo mejores incrementos en la rentabilidad del patrimonio, margen operacional de utilidad, margen bruto de utilidad y, generando mejores estrategias para obtener excelentes resultados.

Lumpkin Eisner, (2011), La inteligencia competitiva ayuda a las empresas a definir y conocer la industria, así como a identificar las fortalezas y debilidades de sus rivales, lo que incluye actividades de inteligencia para reunir datos sobre los competidores e interpretarlo; si se hace correctamente, ayuda a la compañía a evitar sorpresas porque se anticipan los movimientos de los competidores y se disminuye el tiempo de respuesta. (Pág.40)

\subsection{Estrategia de inteligencia competitiva.}

La estrategia de la inteligencia competitiva produce que se incremente la economía empresarial; la situación económica de cada individuo que pertenezca a dicha organización, debido a que creatividad de ideas permiten la innovación de productos involucrando a la tecnología que permite la agilidad de los manejos de la producción.

Lumpkin Eisner, (2011) La empresa casi nunca tiene capacidad para prever las tendencias los hechos que sucederán en el entorno general e incluso menos para controlarlos. (Pág.45).

El entorno como factor de incidencia en la inteligencia competitiva afecta a la empresa, dado a que no se puede conocer todos los objetivos que tienen los factores externos por lo cual se debe analizar los segmentos del entorno.

Las empresas que poseen capacidad de emplear la inteligencia competitiva contienen una planificación estructurada, es decir que puede definir nuevas maneras de hacer negocios, innovar productos, construir mejores habilidades.

\subsection{Competitividad empresarial.}

Sergio Hernandez y Rodriguez, (2011) "La Competitividad de una empresa consiste en crear, desarrollar y sostener capacidades para conquistar la preferencia de los consumidores en los mercados de productos o servicios, y así generar resultados financieros". Pág. 12.

Las empresas operan en un ambiente de libre competencia lo que las obliga a tener productos y servicios que compitan con ventajas diferenciadoras para que los consumidores, o parte de ellos, las selecciones o elijan al comprar. La falta de innovación en estrategias competitivas de las empresas entorpece el crecimiento económico, por falta de visión no existen cambios en el proceso de producción o en la intervención de nuevos productos. Las empresas deben operar en un ambiente de competencia lo que las obligan a tener productos y servicios que compitan con ventajas diferenciadas.

\subsection{Organización de la toma de decisiones.}

Esteban García Canal, (2007) Identificar líneas de actuación para gestionar retos y problemas atraves organizativos que conlleva el crecimiento asociado a las inversiones, a su vez relacionarse con temas de control y supervisión dentro de la organización o empresa, por lo que también tendrían efecto sobre la eficiencia interna, aspecto que ya ha abordado en el presente estudio. Pag.85

La toma de decisiones no es un tema fácil de explicar a la hora de su empleo abarca muchos factores que influyen en la toma de decisiones es un tema muy extenso en la empresa, por esa razón se debe considerar todas las opciones, para así tomar la opción más adecuada.

Es importante que las empresas determinen toma de decisiones asertivas, de tal manera que disminuya los riesgos y amenazas del entorno, y se potencie oportunidades y fortalezas, por ende debería emplear el siguiente proceso:

\section{ILUSTRACIÓN No.- 1 Proceso de toma de decisiones} de manera idóneo.

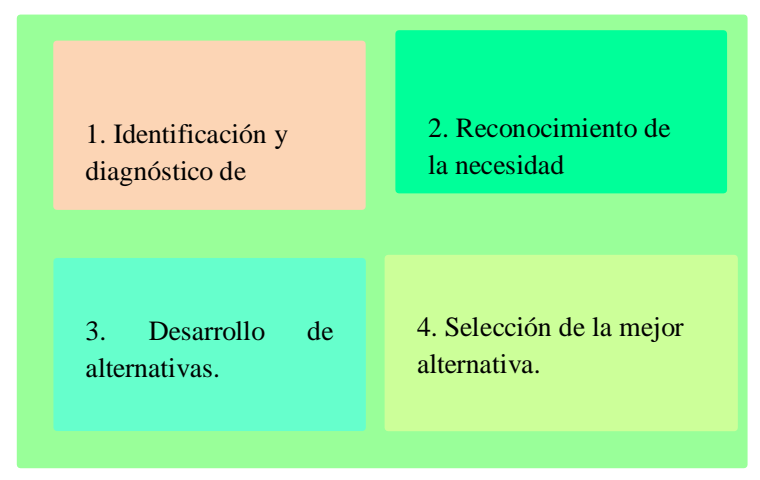

Fuente: Toma de Decisiones García Canal

\subsection{Efectividad administrativa.}


Whetten David A. (2005) identificó siete prácticas fundamentales asociadas con la efectividad administrativa y organizativa: afirmar la seguridad del empleo, contratar selectivamente a la gente, fomentar la descentralización y los equipos autoadministrados, instituir niveles altos de paga basados en el desempeño, capacitar ampliamente, reducir las diferencias de estatus y compartir información."

La efectividad administrativa es el procedimiento que aplican las organizaciones o empresas para poder realizar un estudio en el que recopilen información que permita establecer el entorno de la organización, con el único propósito de validar que los procedimientos internos que se han realizado se han cumplido acertadamente porque están dentro los objetivos principales de la organización y poder incrementar planes de acción orientados a mejorar su efectividad de nuestros objetivos.

En base a las condiciones del entorno las empresas deben emplear estrategias competitivas, que permitan mejorar el crecimiento económico; sin embargo, no realizan estrategias idóneas para consolidar el producto dentro del mercado, y por ende el consumidor no estará satisfecho.

\subsection{Habilidad gerencial.}

Bonache J. \& Cabrera A. , (2002) El manejo de las competencias por parte del gerente contribuiría al cumplimiento de las funciones de la organización, dotando a ésta del personal más competente, puesto que los recursos humanos son particularmente importantes en entornos dinámicos; y es aquí donde los empleados si son adecuadamente gestionados, facilitan la adaptación y renovación continua de la empresa para adecuarla a las demandas del mercado.

Los gerentes son los encargados de dirigir la empresa, hacia un mejor desarrollo empezando por seleccionar el personal idóneo, capacitado para el aporte y crecimiento en cada una de sus áreas, considerando para su selección la capacidad, innovación, experiencia, aplicación de estrategias, que permitan obtener los objetivos planteados. El trabajo en equipo es uno de los ejes fundamentales en el crecimiento y desarrollo de una empresa en el área de producción, existen habilidades que muchas veces no son desarrolladas de forma adecuada por la poca importancia de los directivos y se maneja de forma intelectual.

\subsection{Habilidades de la Gerencia: centro de la estrategia competitiva.}

Fernandez, Cuberio, \& Datziel, (1996) La gerencia de hoy espera que el individuo sea capaz de incorporar sus conocimientos y habilidad a los

N. Collins/S. Linzán procesos desarrollados en la empresa, con lo cual se pretende descubrir las habilidades y conocimientos demostrables, que llevan al logro exitoso de los objetivos organizacionales.

Habitualmente la labor gerencial conlleva a una serie de contexto y herramientas complejas que los directivos deben aplicar de forma eficiente en su labor cotidiana, considerando que a través de sus capacidades y conocimiento van a realizar gestiones innovadoras que contribuyan y fortalezcan el progreso de una empresa. Una de las competencias claves en las empresas es la toma de decisiones frente a los múltiples acontecimientos que se presentan, donde los directivos deben hacer frente a estas situaciones, que en ocasiones se tornan complejas por las exigencias, o por la gran competitividad que existe en el mercado.

\subsection{Objetivo general.}

Evaluar la incidencia de la inteligencia competitiva en el desarrollo económico de las empresas de la provincia de Santa Elena.

\section{Materiales y métodos.}

En la presente investigación se emplearon diferentes métodos de naturaleza práctica y experimental, para conocer sobre la situación actual de las empresas por ello se detalla a continuación los métodos a utilizar: El método inductivo que permitió conocer acerca del objeto de estudio mediante la observación directa permitiendo determinar de forma efectiva características relevantes e importantes.

Para obtener información de fuente primaria se diseñó una entrevista realizada a 32 empresas de la provincia de Santa Elena, con la finalidad de establecer criterios respecto a la inteligencia competitiva y su influencia en el desarrollo económico, mediante un muestreo no probabilístico en el que se eligió a las empresas en base al criterio del investigador.

\subsection{Procesamiento y análisis.}

La información se procesó en función de las técnicas utilizada en la investigación. En lo que se refiere a la tabulación, se utilizó el programa estadístico SPSS 10.1 .

\section{Resultados.}

\subsection{La inteligencia competitiva como factor de productividad.}

La mayor parte de las empresas encuestadas en la provincia de Santa Elena no emplean estrategias de inteligencia competitiva, por ello no detectan 
oportunidades del entorno, ni evalúan el desempeño organizacional, por ende no se potencia ventajas competitivas que contribuyan a obtener mayor productividad e ingresos económicos.

Las decisiones empresariales en las empresas de la provincia de Santa Elena son compartidas por varias personas, debido que las empresas locales tienen una estructura organizacional de tipo familiar, por tanto, toda la información debe llegar a ellas, independientemente de que sean varias o una persona las que se dedican a recoger, procesar, analizar.

\section{ILUSTRACIÓN No.- 2 Pilares de la inteligencia} competitiva

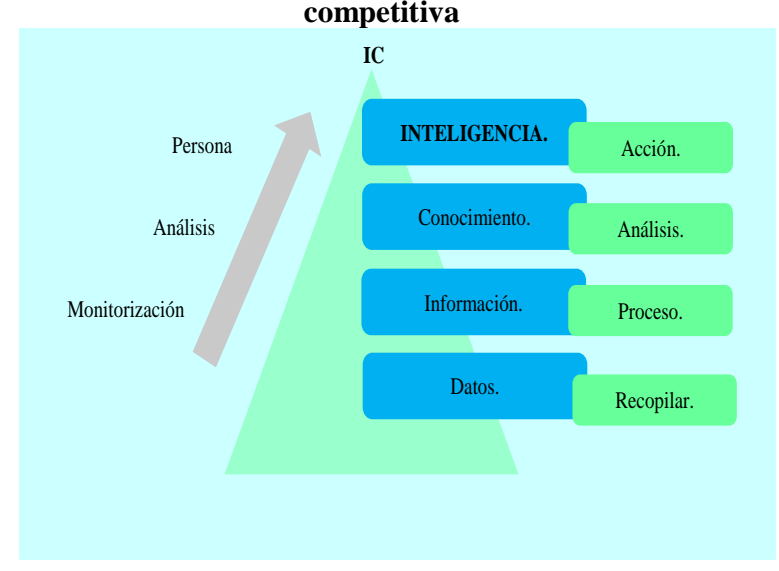

Fuente: Encuesta a empresas

Es importante que las empresas de la provincia de Santa Elena empleen estrategias de inteligencia que permita la recolección de información sobre: los competidores, para su posterior comprensión, clasificación y análisis, a fin de entender las estrategias del mercado empresarial y diseñar novedosas alternativas que permitan solucionar problemas, abarcar nuevos mercados o superar a los competidores, con el objeto de seguir el rumbo apropiado para la empresa, en el momento oportuno.

\subsection{La toma de decisiones como factor de disminución en incertidumbre.}

Para que una empresa sea productiva deberá enfrentar problemas y estos a su vez permitirán tomar decisiones más efectivas, siempre y cuando la empresa tenga buenos empleados y colaboradores que estén comprometidos con la empresa para así ayudar al gerente a tomar decisiones honestas, ya que en ocasiones son los empleados quienes tienen la solución al problema.

Las empresas deben consideran en la toma de decisiones la capacidad y desempeño del personal, la atención que se brinda al cliente se basa en los principios de calidad y respeto. Sin embargo, en las empresas de la provincia de Santa Elena no existe un modelo de gestión administrativa sólido que disminuya el impacto negativo por amenazas del entorno, por ende debería las empresas emplear las siguientes acciones:

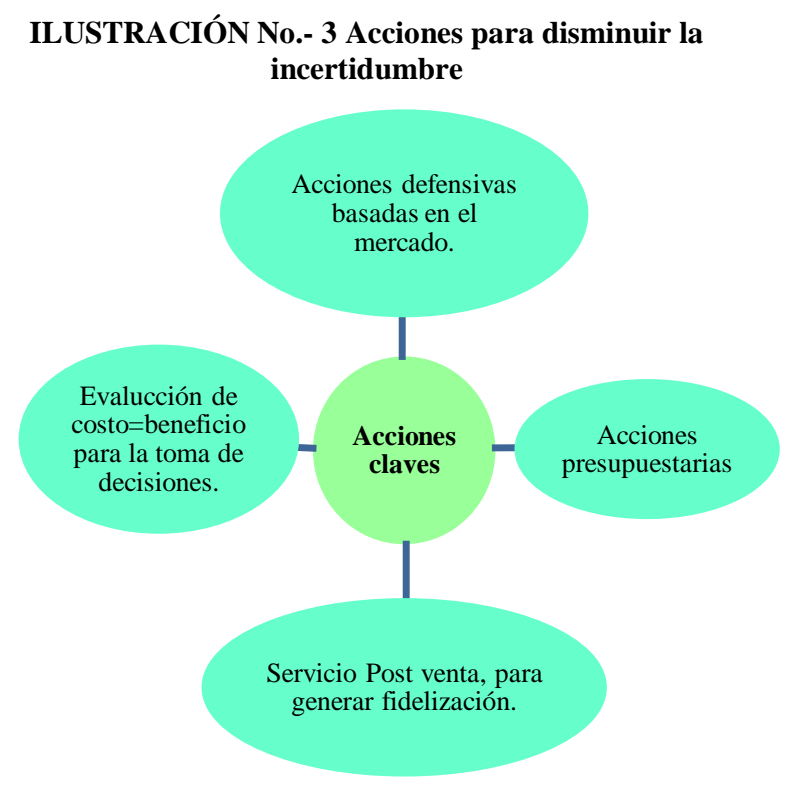

Fuente: Encuesta a empresas

Las empresas de la provincia de Santa Elena deben fortalecer su nivel de competitividad en el mercado para que su empresa crezca, sino también una empresa generando competitividad nacional e internacionalmente dejando así en alto el nombre de nuestra provincia y de nuestro país.

\subsection{La toma de decisiones como factor de disminución de la incertidumbre.}

Las empresas deben realizar la toma de decisiones en base a criterios de los directivos, quienes evalúan los beneficios que se obtendrán por emplear acciones o estrategias; sin embargo, en base a los riesgos del entorno, la ejecución de proyectos y actividades se ven afectadas negativamente; debido al desconocimiento de un proceso adecuado para disminuir la incertidumbre.

La mayor parte de empresas encuestadas consideran importante realizar un proceso de evaluación costooportunidad para la toma de decisiones, en que se debe considerar los beneficios por la implementación de acciones estratégicas.

\section{ILUSTRACIÓN No.- 4 Importancia del proceso de} toma de decisiones 

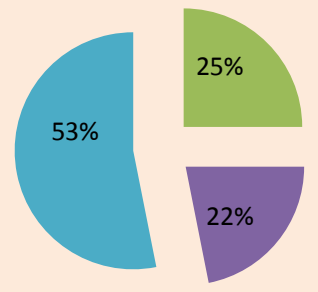

Totalmente

desacuerdo

En desacuerdo

Indiferente

De acuerdo

Fuente: Encuesta a empresas

Las empresas de la provincia de Santa Elena, deberían emplear acciones estratégicas basadas en inteligencia competitiva, que permitan maximizar las oportunidades del entorno generando mejores estrategias para obtener excelentes resultados, con el fin de alcanzar el desarrollo económico empresarial esperado.

\section{Discusión de resultados.}

\subsection{Competitividad empresarial.}

Sergio Hernandez \& Rodriguez, (2011) "La competitividad de una empresa consiste en crear, desarrollar y sostener capacidades para conquistar la preferencia de los consumidores en los mercados de productos o servicios, y así generar resultados financieros”. Pág. 12.

Es importante que las empresas determinen toma de decisiones asertivas, de tal manera que disminuya los riesgos y amenazas del entorno, y se potencie oportunidades y fortalezas.

La competitividad de una empresa consiste en desarrollar nuevas ideas para así deleitar a los consumidores en los mercados, y por medio de estas estrategias poder ver resultados económicamente favorables; sin embargo, lamentablemente en la provincia de Santa Elena, hay empresas que no cuentan con un grupo innovador o creador de nuevas ideas que puedan generar competitividad, ya esto no permite de que las empresas cautiven a los consumidores del mercado dejando así perder clientela y llamando la atención de nuevas personas, y por ende no obtendrán buenos resultados.

\subsection{Toma de decisiones empresariales.}

Jairo A. , (2010) La toma de decisiones es una de las competencias claves para todo ejecutivo así como lo es el aspecto personal de cada uno de los seres humanos, pues son los ejecutivos los responsables de selecciones una entre varias opciones en la empresa.

Una de las competencias claves en las empresas de la provincia de Santa Elena es la toma de decisiones frente a los múltiples acontecimientos que se presentan, donde los directivos deben hacer frente a estas situaciones, que en ocasiones se tornan complejas por las exigencias, o por la gran competitividad que existe en el mercado.

Mediante el estudio realizado se determinó la importancia de tomar decisiones asertivas, ya que de esta forma se va a lograr el fortalecimiento y crecimiento de la producción.

\section{Conclusiones.}

- Es importante que las empresas empleen de manera oportuna la inteligencia competitiva, con el fin de potenciar el desarrollo económico, logrando un alto nivel empresarial, en base al uso adecuado del conocimiento obtenido por la experiencia.

- La empresas deben realizar una evaluación de desempeño, con el fin de evitar una decadencia en el mercado ante sus competidores; de tal manera que puedan fortalecer su nivel de desarrollo económico aplicando estrategias basadas en la inteligencia competitiva, mejorar así su estándar económico.

- Para que una empresa sea productiva deberá aplicar la toma de decisiones, estableciendo reglas estratégicas con todos los empleados y colaboradores que pertenezcan en aquella organización con la finalidad de contar con gente responsables dedicados a su labor, comprometidos totalmente con la organización, dándole seguridad al gerente.

- Es necesario que las empresas consideren las amenazas y oportunidades del entorno, así como por la capacidad de producción, en el análisis de la toma las decisiones y de esta manera poder reducir los riesgos e incertidumbre, y por ende potenciar la optimización de recursos.

\section{Bibliografía.}

1. Armstrong Y Kotler, G. (2013). Principios de marketing. Bogotá: Ecoe Ediciones.

2. Armstrong, G., Kotler, P., Merino, M. J., Pintado, T., \& Juan, J. M. (2011). Introducción al Marketing (Tercera Edición ed.). Madrid, España: Pearson Educación.

3. Bernal Torres César Augusto. (2010). Metodología de la Investigación: Administración, Economía, Humanoides y Ciencias Sociales. Tercera Edición. 
Pearson Education de Colombia, Ltda, Santa Fe de Bogotá.

4. Bonache J. \& Cabrera A. (2002), Dirección Estratégica de Personas, Madrid: Pearson Education.

5. Corbetta, P. (2007). Metodología y técnicas de investigación social. Madrid: Editorial Mcgraw-Hill/Interamericana de España S.A.

6. Crespo R., Perez C., \& Morillas A. (2012). Fundamentos de Marketing. México: Trillas.

7. Echeverría Cañas. (2009). Fundamentos de marketing práctico. México: Pearson.

8. Fernández, Cuberio \& Datziel (1996), Gestión por cometencias, fortalezas, tensiones y paradojas, Madrid Alarcón.

9. García Canal Esteban (2007), Ecoomía Exterior, España

10. Hernández y Rodríguez Sergio (2008), Introducción a la Adminsitración, Teoría general Administrativa, origen, evolución y vanguardia, Mc. Graw Hill
11. Jairo a. (2010), La Gestión por commpetencias ejercicios y coaching empresarial, dos estrategias internas para la organización, Mexico.

12. Lumpkin Eisner, E. (2011). Administración estratégica. México: Mc Graw Hill Educación.

13. Margalef, J. C. (2000). La gestión del crecimiento de la empresa. aravaca. Madrid: Mcgraw-hill/interamericana de españa, s. a. u.

14. Parkin, M., \& Loría, E. (2010). Macroeconomía: Versión para Latinoamérica. México: Pearson Educación.

15. Whetten, David A. 2005, Desarrollo de Habilidades Directivas, Pearson 